\title{
Update on biology and management of mesothelioma
}

\author{
Rachelle Asciak ${ }^{1,2}$, Vineeth George (i) ${ }^{1}$ and Najiib M. Rahman ${ }^{1}$ \\ Number 2 in the Series "Thoracic oncology" \\ Edited by Rudolf Huber and Peter Dorfmüller
}

Affiliations: ${ }^{1}$ Oxford Centre for Respiratory Medicine, University Hospitals NHS Foundation Trust, Oxford, UK. ${ }^{2}$ Mater Dei Hospital, Msida, Malta.

Correspondence: Rachelle Asciak, Oxford Centre for Respiratory Medicine, Oxford University Hospitals NHS Trust, Churchill Hospital, Oxford, OX3 7LE, UK. E-mail: rachelle.asciakdouh.nhs.uk

@ERSpublications

Despite a better understanding of the pathogenesis of malignant pleural mesothelioma, effective treatment that prolongs survival significantly is lacking. Ongoing research may identify select patient groups that may benefit from surgical treatment. https://bit.ly/3k16J9O

Cite this article as: Asciak R, George V, Rahman NM. Update on biology and management of mesothelioma. Eur Respir Rev 2021; 30: 200226 [https://doi.org/10.1183/16000617.0226-2020].

ABSTRACT Malignant pleural mesothelioma is an aggressive, incurable cancer that is usually caused by asbestos exposure several decades before symptoms arise. Despite widespread prohibition of asbestos production and supply, its incidence continues to increase. It is heterogeneous in its presentation and behaviour, and diagnosis can be notoriously difficult. Identification of actionable gene mutations has proven challenging and current treatment options are largely ineffective, with a median survival of 10-12 months. However, the past few years have witnessed major advances in our understanding of the biology and pathogenesis of mesothelioma. This has also revealed the limitations of existing diagnostic algorithms and identified new treatment targets.

Recent clinical trials have re-examined the role of surgery, provided new options for the management of associated pleural effusions and heralded the addition of targeted therapies. The increasing complexity of mesothelioma management, along with a desperate need for further research, means that a multidisciplinary team framework is essential for the delivery of contemporary mesothelioma care.

This review provides a synthesised overview of the current state of knowledge and an update on the latest research in the field.

\section{Introduction}

Malignant pleural mesothelioma (MPM) is an aggressive and incurable malignancy that originates from the mesothelial cells that form the serosal lining of the pleural cavity. The median age at diagnosis is 75 years, and overall survival is $38 \%$ at 1 year and $7 \%$ at 3 years, reflecting the poor prognosis [1]. Despite the increasing prohibition of asbestos production and supply over the past 30-40 years, deaths from MPM continue to rise [2].

Histologically, there are three types of mesothelioma: epithelioid ( 60\%), sarcomatoid (20\%) and biphasic (20\%), which has both epithelioid and sarcomatoid features [3], with median survival of 19, 4

Provenance: Commissioned article, peer reviewed.

Previous articles in this series: No. 1: Eichhorn F, Winter H. How to handle oligometastatic disease in nonsmall cell lung cancer. Eur Respir Rev 30: 2021; 200234.

Received: 10 July 2020 | Accepted after revision: 27 Oct 2020

Copyright CERS 2021. This article is open access and distributed under the terms of the Creative Commons Attribution Non-Commercial Licence 4.0. 
and 12 months, respectively, in a database of 1183 patients with mesothelioma [4]. Treatment with chemotherapy doublet cisplatin/pemetrexed can extend life by $\sim 2-3$ months, with longer median time to progressive disease (6 versus 4 months). Response rates to cisplatin/pemetrexed were $41 \%$ in clinical trial settings [5], but $26.3 \%$ in a subsequent nonrandomised study of 1704 patients [6]. Where licensed, bevacizumab is added to this regime since cisplatin/pemetrexed/bevacizumab combination was associated with median (95\% CI) survival of 19 (16-22) months, versus 16 (14-18) months with cisplatin/ pemetrexed alone [7], with grade 3-4 adverse events occurring in $71 \%$ versus $62 \%$, respectively [7]. A number of patients will not be fit enough to receive first-line chemotherapy, and in addition, of those who do receive chemotherapy, many will risk the associated adverse effects of chemotherapy without any benefit in terms of disease response. It would be ideal to predict the response or resistance to cancer treatment beforehand, so that patients who are not likely to respond to treatment may be spared the associated side-effects.

The mechanism of carcinogenesis is not fully understood, but most cases are attributable to asbestos fibre exposure and inhalation. The pathogenesis of MPM is thought to be multifactorial, and targeted therapies have often proved to be unsuccessful in MPM. Therefore, a better understanding of the biology and pathogenesis of mesothelioma may enable identification of effective treatment targets, and facilitate the development of a personalised treatment approach.

\section{The biology of mesothelioma \\ Pathogenesis \\ Asbestos}

Mesothelioma was first characterised in 1931, but only linked to asbestos in a South African epidemiological study published in 1960 [8]. It is now recognised that $85 \%$ of mesothelioma in males is attributable to occupational exposure to asbestos, but only up to $10 \%$ of people with occupational exposure to asbestos develop mesothelioma $[8,9]$. Asbestos exposure is also linked to lung cancer, and combined cigarette smoking with asbestos exposure increases lung cancer risk from 10- to almost 100-fold compared to unexposed people [10]. In contrast, cigarette smoking does not appear to be associated with increased risk of mesothelioma [11].

Asbestos had been used previously for pottery, but mass mining began in the 20th century when asbestos was used to insulate against heat, fire and corrosion [10]. Some of the high-risk occupations include mechanics working on brake and clutch linings, construction and demolition workers, dockyard and shipyard workers, plumbers and electricians. Nonoccupational exposure may occur via exposure through a household asbestos worker, and living near an asbestos factory. The use of asbestos was banned in the United Kingdom (UK) in 1999, and in all European Union countries in 2005, although compliance with this directive has not been verified in some countries. Due to the long latency period of 30-40 years between first exposure and the development of mesothelioma [12], new mesothelioma cases are still diagnosed yearly. In addition, the inhabitants of countries that have banned asbestos correspond to $16 \%$ of the world population, and therefore currently the incidence of MPM is still increasing worldwide [10]. In an epidemiology study published in 2014, the UK, the Netherlands, Malta and Belgium had the highest rates of mesothelioma in Europe [13]. In the UK, 84\% of patients diagnosed with mesothelioma were male and their cases were probably linked to occupational exposure [1].

Documenting and quantifying asbestos exposure is difficult, because it may not be recalled, may be recalled erroneously or recall may be motivated by the possibility of compensatory damages being awarded. Furthermore, workers within the same workplace may be exposed to varying quantities and types of asbestos. Nonoccupational exposure to asbestos may be present. There is high incidence of mesothelioma in some families, where exposure to asbestos may be through the clothing of a family member who is an asbestos worker, although the possibility that there is a genetic predisposition to mesothelioma has also been suggested, as discussed later [14, 15].

There are two main types of asbestos fibres: amphiboles (straight, long fibres, including amosite (brown asbestos), crocidolite (blue asbestos), anthophyllite and tremolite), and the more commonly used serpentine (short, curly fibres), mainly chrysotile (white asbestos). Exposure to amphiboles carries a higher risk of mesothelioma than chrysotile $[16,17]$. Accessibility of amphiboles to the peripheral lung is higher, and biopersistence of amphiboles was noted in both animal and human studies with continuously increasing levels of recoverable lung amphibole fibre levels, whereas chrysotile fibres are able to be partially digested and cleared from the lungs within months [18]. However, many patients will have been exposed to both amphibole and chrysotile asbestos [14].

Inhaled asbestos fibres reach the parietal pleura via the alveoli, visceral pleura and across the pleural space, or via retrograde flow through the lymphatic vessels. Chronic inflammation precedes mesothelioma, and is 
mostly found around stomata and lymphoid patches in the basal parietal pleura, which is the most common site for mesothelioma [10]. MPM progresses from the parietal to the visceral pleura and invades surrounding tissues, with involvement of the visceral pleura denoting more advanced disease (higher stage on the eighth version of the International Association for the Study of Lung Cancer (IASLC) staging system) [19].

The pathogenesis of mesothelioma is multifactorial. Crocidolite asbestos fibres induce toxicity in human mesothelial cells in vitro in a dose-dependent manner [20]. This raises the question of how MPM develops if the asbestos fibres kill the mesothelial cells. Crocidolite has been shown to induce macrophage accumulation within pleura and lung. Macrophages release tumour necrosis factor (TNF)- $\alpha$ upon contact with crocidolite, and human mesothelial cells express TNF- $\alpha$ receptors. Additionally, asbestos causes the human mesothelial cells to secrete TNF- $\alpha$. The TNF- $\alpha$ ligand-receptor interaction activates the NF- $\kappa \mathrm{B}$ pathway, which allows the human mesothelial cells to divide rather than die, acquiring resistance to apoptosis [21]. If there is sufficient DNA abnormality caused by asbestos, then the mesothelial cells may develop into mesothelioma. Exposure to asbestos is thought to trigger several processes that ultimately lead to mesothelioma, which include the following.

1. Inhaled fibres cause irritation with subsequent repeated cycles of tissue damage and repair and local inflammation.

2. Direct physical interaction: asbestos fibres penetrate mesothelial cells and disrupt mitotic spindles resulting in chromosomal abnormalities.

3. Mesothelial cells exposed to asbestos release inflammatory cytokines and growth factors, inducing inflammation and creating a pro-tumour microenvironment.

4. Macrophages phagocytose asbestos fibres, but their inability to digest them results in the production of reactive oxygen species, leading to intracellular DNA damage and abnormal repair.

5. Mesothelial cell death as a result of asbestos leads to release of high mobility group box 1, which further promotes and sustains chronic inflammation.

6. Phosphorylation of protein kinases leads to increased expression of proto-oncogenes, promoting abnormal cellular proliferation [22-24].

\section{Other factors}

The majority of workers exposed to asbestos do not develop mesothelioma, and $20 \%$ of cases of mesothelioma are not recognised to be associated with asbestos exposure [25]. In addition, mesothelioma develops in adults after a very long latency period, and there are rare cases of mesothelioma that are diagnosed in children [26], implying that mesothelioma in children either behaves very differently from the mesothelioma in adults, or is not associated with asbestos exposure in the same way. These findings suggest that other factors, apart from asbestos, are involved.

Simian virus 40

Simian virus 40 (SV40), a DNA tumour virus, is endogenous to the rhesus monkey, and is thought to have infected humans via contaminated polio vaccines in the 1950s and 1960s [27, 28], although SV40 was also detected in people who had not received the vaccine, indicating that other routes of infection exist [2, 22, 23].

SV40-like DNA sequences were found in up to $60 \%$ of human mesothelioma specimens in France and USA $[29,30]$, and similar results were also reported in an independent multilaboratory USA study (10 (83\%) out of 12 mesotheliomas were found to have SV40 DNA sequences) and in several other laboratories worldwide $[31,32]$. Furthermore, $>50 \%$ of hamsters injected with SV40 developed mesothelioma [33]. In addition, human mesothelial cells were found to be more susceptible to SV40 infection and transformation than fibroblasts and epithelial cells, with SV40 and asbestos found to be co-carcinogenic in vitro [20]. Conversely, SV40 was not found in Turkish $(n=9)$ or Finnish $(n=49)$ mesothelioma samples [34, 35], possibly related to geographical differences in distribution of administration of SV40-contaminated vaccines.

However, it has been suggested that in the above studies, the SV40 DNA sequences documented to have been found in mesothelioma represent false positives from contaminating plasmids resulting in positive sequencing results [36]. However, this would not explain the positive results in animal models. Consequently, the role of SV40 in mesothelioma remains controversial.

\section{Radiation}

Ionising radiation has been identified as a human carcinogen and risk factor for several cancer types. Cases of the development of mesothelioma after radiation exposure have been reported, with two epidemiological studies showing increased risk of mesothelioma after radiation for testicular cancer (relative risk 4, 95\% CI 2-8.1), and lymphoma (observed/expected ratio 2.3, 95\% CI 1-4.3) [37-39]. 
Erionite

The high incidence of mesothelioma in Karain and Tuzkoy villages in Turkey is attributed to exposure to erionite, a mineral fibre commonly found in the stones of the houses in these villages [14, 40, 41]. Mineralogical and pedigree analysis in these villages revealed that there may also be an autosomal dominant genetic susceptibility to mesothelioma [42]. In addition, there is evidence from animal models that erionite is a more potent inducer of MPM than asbestos, with 40 (100\%) out of 40 of rats injected with intrapleural erionite developing MPM, compared to 19 (47.5\%) out of 40 of rats injected with intrapleural asbestos [43].

\section{Genetic mutations in mesothelioma}

The identification of a specific gene mutation in mesothelioma would aid the development of targeted therapy. However, mesothelioma is notoriously heterogeneous in its presentation, behaviour and aggressiveness, and its genetic make-up is no exception: a specific genetic mutation common to all mesothelioma has not been identified. DNA sequencing of 198 unrelated patients with mesothelioma revealed that at least one germline mutation was present in $12 \%$, and $25 \%$ of the mutations detected were in BAP1, $12.5 \%$ in BRCA2 and $8.3 \%$ in CDKN2A (the odds of having a mutation OR 1658, 95\% CI 19976224, p<0.001; OR 5, 95\% CI 1-14.7, p 0.03; OR 53, 95\% CI 6-249, p<0.001, respectively, compared with the noncancer control population) [44].

The genes identified as being commonly mutated in MPM are those in the p53/DNA repair pathway (TP53, SMARCB1, BAP1) and PI3K-AKT pathway (PDGFRA, KIT, KDR, HRAS, PIK3CA, STK11, NF2), with the commonest being in BAP1, CDKN2A and NF2 [45-50]. In contrast, K-ras activation is rare in mesothelioma, suggesting that development of mesothelioma is not dependent on this pathway [51]. The interpatient variation of the mutational landscape for these genes highlights the potential importance of personalised and targeted therapies in MPM.

The tumour suppressor genes exhibited nonsynonymous, stop codon gain and frameshift insertion mutations as identified by integrated genomic studies on MPM tumour samples [48]. The tumour suppressor gene TP53 plays a central role in the response to DNA damage and induces cell apoptosis in cells with DNA damage. Mutations in TP53 occur in $>50 \%$ of all human cancers [52], and in $\sim 5-8 \%$ of MPM [53, 54]. Mutated TP53 is associated with significantly decreased survival in MPM compared with wild-type TP53 [54]. Other frequent mutations in MPM are in the tumour suppressor NF2 in 50\%, and these are associated with increased proliferation and invasiveness of mesothelioma; and conversely, BAP1 loss is present in $\sim 30-60 \%$ of MPM and is associated with improved prognosis [46, 55]. In addition, loss of INK4A/Arf in mice with MPM was associated with significantly reduced median survival and highly invasive tumours, suggesting that INK4A loss substantially contributes to the poor clinical outcome of MPM [56]. Mutations in NF2 and INK4A, genes involved in apoptosis regulation, may explain, at least in part, the resistance of mesothelioma to most conventional drugs, because the cells are resistant to the induction of apoptosis.

Mesothelioma ( $\mathrm{n}=106$ mesothelioma samples in total) had the lowest percentage of samples with an actionable mutation out of 10945 tumour samples of varied primary malignancies. Those mesothelioma samples that did have actionable mutations had the lowest level of actionability based on the identified mutation [50]. These studies further highlight the uphill struggle to identify clinically useful, more targeted therapies for MPM despite the use of advanced techniques.

\section{The management of mesothelioma}

In patients with MPM, the onset of disease is often insidious and the diagnosis difficult to make. Patients predominantly present with dyspnoea, chest pain or both, and can often receive treatment for other benign conditions prior to the diagnosis being made $[2,12]$.

Current treatment options are relatively ineffective and median survival is 10-12 months [57]. However, early diagnosis in a multidisciplinary framework is key to limiting morbidity and prolonging survival.

\section{Pathological confirmation}

The diagnosis of MPM depends on positive pleural fluid cytology or histological confirmation via pleural biopsy [12]. Existing guidelines recommend thoracocentesis as the initial pleural procedure, and sensitivities as high as $73 \%$ have been reported based on audit data from single centres [12, 58]. However, more recent prospective studies have demonstrated that the diagnostic yield of pleural fluid cytology can be as low as $0-6 \%$ for MPM $[59,60]$.

Consequently, pleural biopsies with appropriate immunohistochemistry are required for the vast majority of cases $[12,61]$. These can be obtained percutaneously under radiological guidance or under direct vision at 
thoracoscopy. Thoracoscopic biopsies from multiple sites remain the preferred approach, as the larger biopsy size generally increases diagnostic confidence and allows for histological subtyping [62]. In addition, deeper biopsies allow for the assessment of tumour invasion by including fat and/or skeletal muscle [63].

Image-guided biopsies have been shown to be effective in diagnosing malignant pleural disease [64-66]. However, there are limited data on their use specifically for mesothelioma and current guidelines highlight that they are of particular value for patients who are unable to tolerate thoracoscopy [12].

More recently, confocal laser endomicroscopy (CLE) has been used to provide real-time imaging at a cellular level. This technology has been used in other areas of medicine to increase diagnostic yield by differentiating malignant tissue from benign fibrosis. A recent feasibility study of 20 patients with suspected MPM suggested that fluorescein-aided CLE could be used to differentiate epithelioid and sarcomatoid MPM from pleural fibrosis regardless of the biopsy method [67].

The recently concluded TARGET trial randomised patients who had an initial nondiagnostic computed tomography (CT)-guided percutaneous pleural biopsy to either a repeat procedure or a pleural biopsy from areas of high 2-fluoro-2-deoxy-D-glucose uptake on CT-positron emission tomography (PET). Data presented at the recent British Thoracic Society Winter Meeting demonstrated that there was no significant difference in sensitivity for the diagnosis of MPM with the latter approach compared to standard CT-guided biopsy [68]. However, the full results are keenly awaited.

\section{Staging}

A number of staging systems have been used for MPM [69]. The eighth edition of the IASLC tumour, node, metastasis (TNM) staging system, which was published in 2018, was developed using an international cohort study with data on almost 2000 patients with pathologically confirmed MPM [19]. Disease stage using the TNM classification was shown to be a key predictor of survival in this population and this is now the preferred system for MPM staging $[12,62]$.

A number of imaging modalities have been evaluated for the staging of MPM. However, all of these compare unfavourably to operative staging with mediastinoscopy to assess lymph node involvement [12].

CT (figure 1) of the chest and abdomen is considered the primary cross-sectional imaging modality and is the mainstay of radiological staging. It is both widely used and easily accessible. However, it performs poorly when assessing locoregional soft tissue disease or nodal metastases [12]. Contrast-enhanced magnetic resonance imaging (MRI) (figure 2) is better to evaluate chest wall and diaphragmatic invasion. One prospective case series of 76 patients who underwent radical surgery correlated radiological staging with final pathological staging [70]. In this setting, MRI revealed the presence of 17 (22\%) unresectable T4 or M1 tumours which were not identified by CT. However, over half the cases in this series were pathologically upstaged due to more advanced pericardial involvement, which was evident intraoperatively. Furthermore, the accuracy of MRI in assessing nodal disease was low with a reported sensitivity of $66 \%$ for assessing disease at stage $\mathrm{N} 2$ or less.

CT-PET has been shown to be superior to CT in identifying extrathoracic disease and is often used for assessing response to therapy $[71,72]$. A small retrospective review suggested CT-PET was superior in

FIGURE 1 Computed tomography scan image of the chest, with right-sided irregular and nodular pleural thickening in a case of malignant pleural mesothelioma.

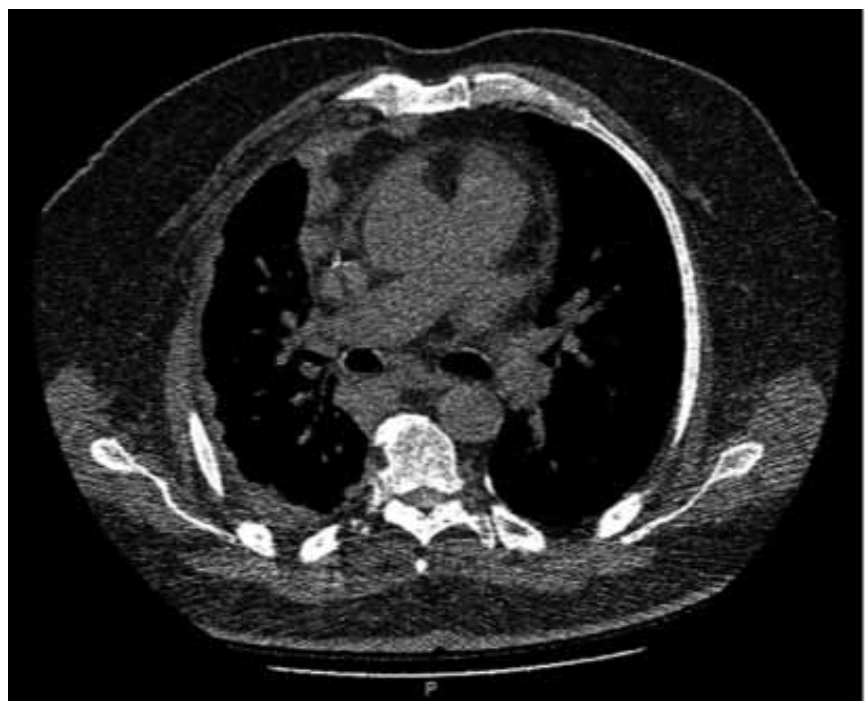



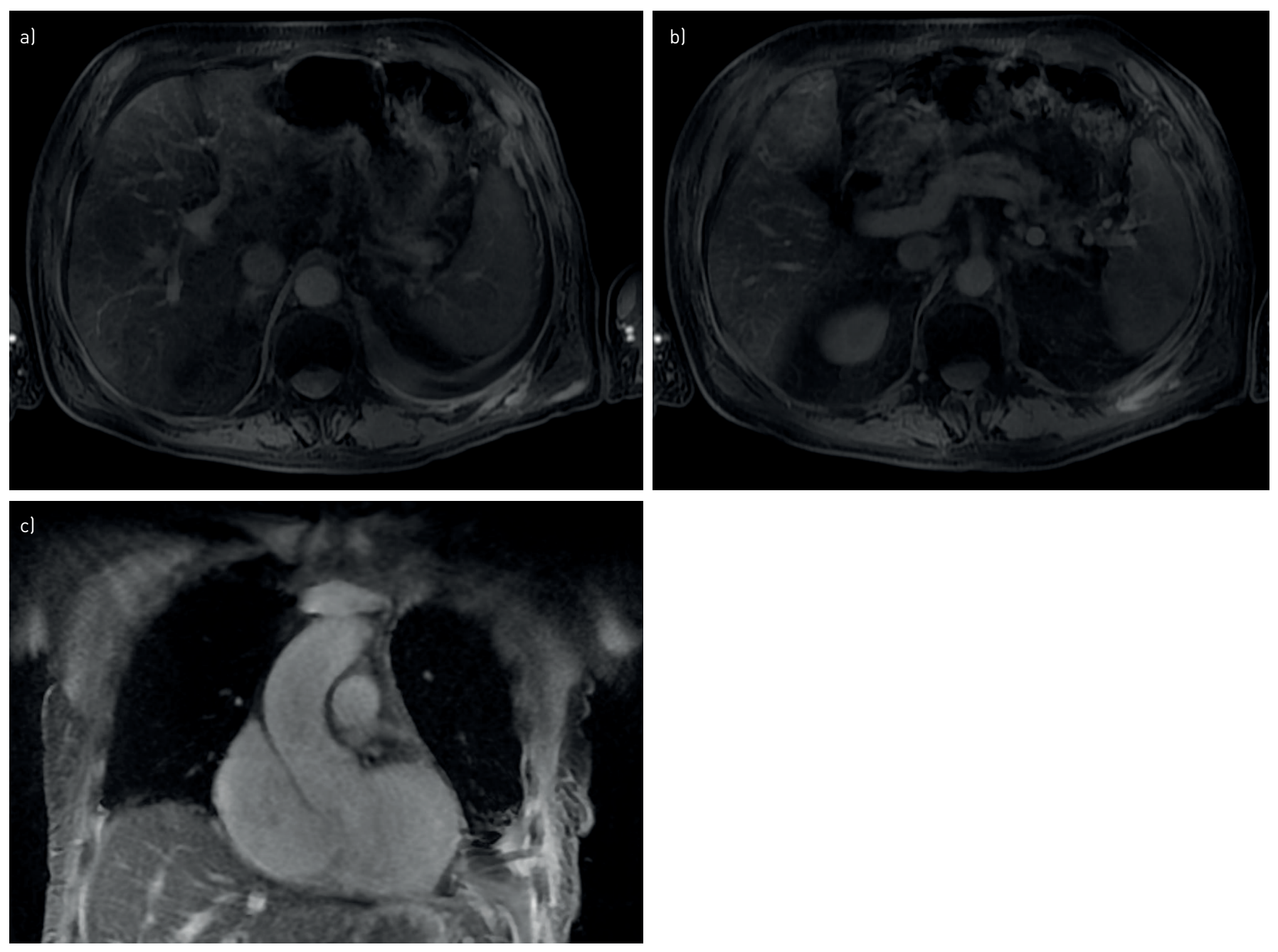

FIGURE 2 Axial magnetic resonance imaging (MRI) thorax images (T1 with contrast) demonstrate a) left-sided irregular pleural thickening and b) left-sided enhancing posterior chest wall invasion in a patient with malignant pleural mesothelioma; c) coronal MRI thorax image of the same patient, demonstrating the left-sided enhancing pleural thickening and chest wall invasion.

identifying nodal disease, with 14 out of 35 individuals upstaged, with a consequent decision not to proceed to surgery [73].

\section{Surgical treatment options}

Surgery is controversial and limited to a small number of patients with early-stage disease. Research into its utility has been hampered by varying definitions of surgical procedures and a heterogeneity of practice. However, the 2011 IASLC International Staging Committee and the International Mesothelioma Interest Group consensus statement offered some clarity by defining procedures into four distinct groups (table 1)

TABLE 1 The four surgical options in malignant pleural mesothelioma, as described by RICE et al. [74]

\section{Extrapleural pneumonectomy \\ Extended pleurectomy decortication \\ Pleurectomy decortication}

Partial pleurectomy
En bloc resection of the parietal and visceral pleura with the ipsilateral lung, +/- pericardium and diaphragm

Parietal and visceral pleurectomy to remove all gross tumour with resection of the diaphragm and/or pericardium

Parietal and visceral pleurectomy to remove all gross tumour without diaphragm or pericardial resection

Partial removal of parietal and/or visceral pleura for diagnostic or palliative purposes, but leaving gross tumour behind 
[74]. These include extrapleural pneumonectomy (EPP) and extended pleurectomy decortication (EPD), which aim to achieve maximal macroscopic resection and are nominally curative, and those such as partial pleurectomy or pleurectomy/decortication, which are performed with purely palliative intent [74].

EPP involves en bloc resection of the parietal and visceral pleura along with the ipsilateral lung, pericardium and hemidiaphragm if necessary. EPD is lung-preserving, but attempts to remove all gross tumour with resection of the parietal and visceral pleura and diaphragm and/or pericardium [74].

Although many guidelines recommend maximal macroscopic resection for patients with early-stage mesothelioma, there is a paucity of high-quality evidence to support this. Both of these procedures are associated with significant post-operative mortality and morbidity, with some studies reporting morality rates as high as $15 \%$ when delivered as part of multimodal therapy [75].

The multicentre Mesothelioma and Radical Surgery (MARS) feasibility trial, which was published in 2011 by Treasure et al. [76] was the first, and, to date, only prospective randomised controlled trial (RCT) to evaluate the efficacy of EPP in addition to chemotherapy alone. Participants were given induction platinum-based chemotherapy with subsequent randomisation to either EPP with subsequent radiotherapy or no EPP. Although a feasibility study by design, it suggested that EPP, as part of a trimodality approach, shortened survival compared to chemotherapy alone and had no effect on quality of life. In addition, there was a high rate of adverse events in the interventional arm, with a post-operative mortality rate of $19 \%$.

Meta-analyses in the past decade have suggested that short-term perioperative mortality is higher in EPP compared to EPD, with a suggestion that long-term survival is also better in the EPD group [77, 78]. Consequently, EPP has largely been abandoned with practice shifting towards the less aggressive EPD [2, 79].

However, there is little high-quality evidence to support the use of EPD. To date, studies evaluating its use have been observational, with most being single-centre and limited to highly selected patients with early-stage epithelioid disease. The MARS 2 trial (clinicaltrials.gov identifier NCT02040272), which is currently recruiting across the UK, seeks to address this significant gap in the literature by randomising patients to EPD or pleurectomy decortication, at the surgeon's discretion, after chemotherapy induction compared to chemotherapy alone.

Pleurectomy decortication and partial pleurectomy, unlike EPP and EPD, do not attempt macroscopic complete resection and are debulking procedures. Neither has been shown to improve survival compared to no surgery, but they may improve quality of life. A systematic review by ScHWARTZ et al. [80] revealed eight studies (total of 432 patients) which evaluated the effect of pleurectomy decortication or partial pleurectomy on quality of life. These procedures were generally thought to improve symptom control and quality of life; however, only one of these studies had a comparator group and there was a reliance on observational data.

The MesoVATS trial by Rintoul et al. [81] randomised patients to either video-assisted thoracoscopic partial pleurectomy (VATS-PP) or talc pleurodesis via chest drain. This demonstrated that VATS-PP did not confer a survival benefit compared to standard talc pleurodesis, which was its primary outcome. However, analysis of secondary outcomes suggested that quality of life, as measured by the EQ-5D, was significantly better at 6 and 12 months.

Given the lack of robust data backing surgery in MPM, the British Thoracic Society guidelines do not recommend surgery outside clinical trials [12], although the American Society of Clinical Oncology guidelines recommend maximal surgical cytoreduction in selected patients with early-stage nonsarcomatoid MPM, as part of multimodality treatment, but should not be considered for sarcomatoid mesothelioma [61]. The European Respiratory Society/European Society of Thoracic Surgeons/European Association for Cardio-Thoracic Surgery/ European Society for Radiotherapy and Oncology guidelines [62] state that surgery may be appropriate for a subgroup of carefully selected patients, with extended EPD rather than EPP, but that radical surgery is not recommended for sarcomatoid or predominantly sarcomatoid mesothelioma outside clinical trials. The authors' view is that EPP should never be offered for mesothelioma except in highly selected circumstances, and that surgery in general should be offered only in clinical trials where available, and in highly selected patients if trials are not recruiting. One may take the view that the collated data on surgery suggest that less surgery is better.

\section{Systemic anticancer therapy}

The results of chemotherapy have traditionally been thought to be disappointing due to low response rates and the systemic toxicity associated with treatment regimens. However, it is one of the only treatment modalities that has been shown to improve survival in MPM.

Current first-line therapy stems from a 2003 study by Vogelzang et al. [5]. They randomised 456 treatment-naïve patients to cisplatin with pemetrexed or cisplatin alone and demonstrated that combination therapy improved median survival from 9.3 months to 12.1 months ( $p=0.02)$, with 
significantly higher response rates to treatment. A second RCT by VAN MEERBEECK et al. [82] also evaluated the addition of a folate antimetabolite, raltitrexed, with single-agent cisplatin in 2005. This provided similar results with improved median survival, progression-free survival and response rate compared to cisplatin monotherapy. However, the recruitment of this study fell short of the revised accrual target recommended by its independent data monitoring committee.

A nonrandomised open-label study in 2008 evaluated the safety and efficacy of carboplatin in combination with pemetrexed compared to that of cisplatin. Although the data should be interpreted with caution, carboplatin demonstrated a similar response rate and median survival compared to cisplatin [6]. It is generally better tolerated and is now considered an option for patients who are thought to be unfit for cisplatin.

Treatment response in sarcomatoid mesothelioma is poorly reported, but appears to be lower for sarcomatoid mesothelioma, with four (22\%) out of 18 patients with sarcomatoid mesothelioma responding to treatment with cisplatin/pemetrexed combination $[5,83]$.

\section{Second-line therapy}

The evaluation of second-line therapy has largely been limited to phase II trials and the optimal second-line agent is not known. A systematic review by BuikHuisen et al. [84] conducted in 2015 suggested that single-agent vinorelbine or pemetrexed were reasonable options for those who had relapsed on first-line therapy. Only two of the included 10 studies were phase III trials and one of these did not show any benefit in progression or overall survival with the addition of thalidomide. The other, by JASSEM et al. [85], demonstrated that pemetrexed provided improved response rate and disease control compared to best supportive care in patients who had not received prior pemetrexed. No benefit in overall survival was demonstrated; however, the authors hypothesise that this may reflect the use of post-discontinuation chemotherapy in the best supportive care arm.

Given the paucity of data, patients with adequate performance status should be enrolled in clinical trials where possible.

\section{Targeted therapies}

The addition of bevacizumab, a vascular endothelial growth factor inhibitor, to cisplatin and pemetrexed as first-line therapy was evaluated by ZaLCMAN et al. [7] in a randomised, controlled open-label phase 3 trial. 448 individuals with unresectable disease from centres across France were randomised to receive cisplatin and pemetrexed with or without bevacizumab. The addition of bevacizumab extended median survival from 16.1 months to 18.8 months $(\mathrm{p}=0.167)$. A higher rate of adverse events was noted in the bevacizumab group; however, the majority of these were expected, such as higher rates of thrombotic events and hypertension [7].

The past few years have witnessed the publication of data on the utility of immunotherapy in the treatment of mesothelioma. The DREAM study (Durvalumab with First-Line Chemotherapy in Mesothelioma) investigated the addition of the programmed death ligand 1 inhibitor durvalumab to standard-of-care chemotherapy [86]. The primary end-point of progression-free survival was $57 \%$ at 6 months, which is encouraging, but also suggests early escape from immune control. These results have led to an international randomised phase 3 study, which is currently ongoing.

A number of other targeted agents are also being evaluated for use in mesothelioma, but thus far data have largely been limited to small series and results have been modest. The trial CheckMate743 is currently testing whether there is a benefit of combination immunotherapy (nivolumab plus ipilimumab) over standard chemotherapy. Early results presented by Baas et al. at the IASLC World Conference on Lung Cancer in August 2020 were encouraging, and reported that nivolumab and ipilimumab in nonepithelioid mesothelioma resulted in 18.1 months overall survival compared to 8.8 months overall survival with chemotherapy (hazard ratio $0.46,95 \%$ CI 0.31-0.68). Similarly, the UK's CONFIRM study, which will randomise 336 patients with progression to nivolumab or placebo after at least two treatments, is currently recruiting and due to be completed by mid-2021 (ClinicalTrials.gov identifier NCT03063450).

\section{Radiotherapy}

Radiotherapy has been used both for focal palliation and as part of multimodality regimens after cytoreductive surgery to limit disease relapse in the ipsilateral hemithorax. However, there are limited data to support the effectiveness of this approach or its impact on survival [12]

A number of centres have reported extremely encouraging overall survival with adjuvant intensity-modulated radiotherapy following EPP or EPD [87]. However, these case series findings have not been replicated in larger multicentre trials and the authors of these studies have subsequently concluded that the routine use of hemithoracic radiotherapy cannot be justified $[88,89]$. 
Radiotherapy is also used with palliative intent to reduce chest wall masses or alleviate pain associated with rib invasion or infiltration of intercostal nerves. However, the evidence for this has been limited with most of the published data coming from retrospective case series or single-arm prospective studies. Furthermore, responses are often not durable, with studies demonstrating pain recurrence within the previous radiotherapy field and typically within 3 months of treatment [90].

The largest of these studies, the single-arm SYSTEMS study, recruited 40 patients from three UK centres. It reported that a third of patients had a significant improvement in their pain at 12 weeks, with $12.5 \%$ of patients experiencing a complete response [91]. SYSTEMS-2, a multicentre phase II randomised dose escalation study commenced in 2016 and is due to report shortly (isrctn.com identifier ISRCTN12698107).

\section{Control of associated pleural effusions}

The majority of patients with mesothelioma develop a pleural effusion during their disease course [2]. Traditional management has involved admission to hospital and intrapleural administration of sterile talc through a standard chest tube [92]. However, talc pleurodesis has been shown to fail in preventing recurrent effusions at 30 days in $\sim 30 \%$ of cases [93], and patients with MPM have lower rate of talc pleurodesis success compared with nonmesothelioma patients ( $73 \%$ versus $85 \%, \mathrm{p}=0.02)$ [94]. This may be related to higher rates of nonexpandable lung in MPM patients, greater pleural tumour bulk in MPM leading to decreased area of normal pleura available for pleurodesis success. This management paradigm is also associated with increased healthcare costs [95].

At least three large high-quality RCTs have demonstrated that indwelling pleural catheters (IPCs) provide equivalent control of dyspnoea compared to talc pleurodesis and are associated with reduced inpatient stay [96-98]. In a trial comparing IPC with talc pleurodesis, 38 patients with mesothelioma were included, and had IPC insertion $(n=20)$ or talc pleurodesis $(n=18)$ : effusion-related hospital stay was 1 versus 3 days, respectively $(\mathrm{p}=0.003)$ [99]. They are now considered to be effective first-line treatment, in addition to the standard indication of trapped lung $[96,99,100]$. Their use is increasing worldwide, and compared to conventional treatment with talc pleurodesis, IPC insertion is associated with reduced hospital stays and fewer subsequent pleural procedures [99].

However, the burden of IPC use both on patients and health services is often not fully recognised [101]. Rates of nonserious complications are high in most IPC studies and successful use of IPCs is reliant on adequate community follow-up and support [102].

A number of RCTs have explored optimal IPC strategies. These have shown that more aggressive drainage strategies leading to higher rates of pleurodesis, which would facilitate IPC removal, without leading to improved breathlessness [103-105]. Notable among these was the IPC-Plus study by BhatNaGar et al. [105]. This randomised 154 patients ( $n=23$ mesothelioma) to either talc slurry or placebo delivered through the indwelling pleural catheter. They demonstrated that at day 35 the pleurodesis rate in the intervention arm (43\%) was significantly higher than that of the placebo group (23\%).

Successful pleurodesis may be associated with a survival benefit. In a study of patients with MPE undergoing talc pleurodesis, two datasets were analysed, with MPM being the commonest malignancy in both datasets: $22(36.6 \%)$ out of 60 patients in dataset 1 and $104(40 \%)$ out of 259 patients in dataset 2. Pleurodesis success, defined as absence of the need for further therapeutic pleural procedures at 3 months after intrapleural talc administration, was associated with improved survival (adjusted odds ratios for decreased survival with pleurodesis failure 2.9 (95\% CI 1.1-7.5, p=0.03) and 1.6 (95\% CI 1.1-2.4, p=0.02), respectively) [106]. Successful pleurodesis is more likely with talc, especially via a large-bore chest drain, than with an IPC $(\sim 70-75 \%$ versus $51 \%$, respectively $[93,96]$.

\section{Multidisciplinary team care}

The modern management of mesothelioma is delivered through multidisciplinary teams. These draw on skills from across the healthcare spectrum and aim to streamline the often protracted diagnostic and therapeutic pathways that patients experience.

While there is no set template for this, most larger multidisciplinary team meetings will have access to input from pleural teams, thoracic surgery, thoracic radiology, pathology and oncology. Given the outcomes of mesothelioma, palliative care input is crucial to resolve complex physical symptoms, psychosocial or spiritual needs; however, routine early referral does not appear to confer any benefit in health-related quality of life or mood [107].

Mesothelioma specialist nurses have an integral role in providing and coordinating the care needs of patients and their carers. 


\section{Conclusion}

Despite asbestos use being banned in many countries, new cases of MPM are still being diagnosed because of the lag between exposure to asbestos and diagnosis of MPM. In addition, although asbestos is the most common factor to be associated with MPM, other factors may also be involved including exposure to erionite and radiation, and genetic factors.

The management of MPM can be challenging because of paucity of effective treatments that prolong survival significantly. Cisplatin and pemetrexed combination, with addition of bevacizumab where available, is first-line treatment, but only in patients with adequate performance status. The outcomes of surgery remain controversial and hopefully, ongoing trials will shed light on whether certain surgical options will be beneficial in select patient groups.

Conflict of interest: R. Asciak has nothing to disclose. V. George reports personal fees from Teva UK Ltd/Haymarket Media Group Ltd, outside the submitted work. N.M. Rahman has nothing to disclose.

\section{References}

1 Royal College of Physicians (RCP). National Mesothelioma Audit report 2018 (audit period 2014-16). London, RCP, 2018. www.rcplondon.ac.uk/projects/outputs/national-mesothelioma-audit-report-2018-audit-period-2014-16 Date last accessed: June 30, 2018

2 Bibby AC, Tsim S, Kanellakis N, et al. Malignant pleural mesothelioma: an update on investigation, diagnosis and treatment. Eur Respir Rev 2016; 25: 472-486.

3 Attanoos RL, Gibbs AR. Pathology of malignant mesothelioma. Histopathology 1997; 30: 403-418.

4 Meyerhoff RR, Yang C-FJ, Speicher PJ, et al. Impact of mesothelioma histologic subtype on outcomes in the Surveillance, Epidemiology, and End Results database. J Surg Res 2015; 196: 23-32.

5 Vogelzang NJ, Rusthoven JJ, Symanowski J, et al. Phase III study of pemetrexed in combination with cisplatin versus cisplatin alone in patients with malignant pleural mesothelioma. J Clin Oncol 2003; 21: 2636-2644.

6 Santoro A, O'Brien ME, Stahel RA, et al. Pemetrexed plus cisplatin or pemetrexed plus carboplatin for chemonaïve patients with malignant pleural mesothelioma: results of the International Expanded Access Program. J Thorac Oncol 2008; 3: 756-763.

7 Zalcman G, Mazieres J, Margery J, et al. Bevacizumab for newly diagnosed pleural mesothelioma in the Mesothelioma Avastin Cisplatin Pemetrexed Study (MAPS): a randomised, controlled, open-label, phase 3 trial. Lancet 2016; 387: 1405-1414.

8 Wagner JC, Sleggs CA, Marchand P. Diffuse pleural mesothelioma and asbestos exposure in the North Western Cape Province. Br J Ind Med 1960; 17: 260-271.

9 Hillerdal G. Mesothelioma: cases associated with non-occupational and low dose exposures. Occup Environ Med 1999; 56: 505-513.

10 Røe OD, Stella GM. Malignant pleural mesothelioma: history, controversy and future of a manmade epidemic. Eur Respir Rev 2015; 24: 115-131.

11 Muscat JE, Wynder EL. Cigarette smoking, asbestos exposure, and malignant mesothelioma. Cancer Res 1991; 51: 2263-2267.

12 Woolhouse I, Bishop L, Darlison L, et al. British Thoracic Society guideline for the investigation and management of malignant pleural mesothelioma. Thorax 2018; 73: Suppl. 1, i1-i30.

13 Bianchi C, Bianchi T. Global mesothelioma epidemic: trend and features. Indian J Occup Environ Med 2014; 18 : $82-88$.

14 Carbone M, Kratzke RA, Testa JR. The pathogenesis of mesothelioma. Semin Oncol 2002; 29: 2-17.

15 Huncharek M. Genetic factors in the aetiology of malignant mesothelioma. Eur J Cancer 1995; 31A: 1741-1747.

16 Gilham C, Rake C, Burdett G, et al. Pleural mesothelioma and lung cancer risks in relation to occupational history and asbestos lung burden. Occup Environ Med 2016; 73: 290-299.

17 Nicholson WJ. Comparative dose-response relationships of asbestos fiber types: magnitudes and uncertainties. Ann NY Acad Sci 1991; 643: 74-84.

18 Churg A. Deposition and clearance of chrysotile asbestos. Ann Occup Hyg 1994; 38: 625-633.

19 Berzenji L, Van Schil PE, Carp L. The eighth TNM classification for malignant pleural mesothelioma. Transl Lung Cancer Res 2018; 7: 543-549.

20 Bocchetta M, Di Resta I, Powers A, et al. Human mesothelial cells are unusually susceptible to simian virus 40-mediated transformation and asbestos cocarcinogenicity. Proc Natl Acad Sci USA 2000; 97: 10214-10219.

21 Yang H, Bocchetta M, Kroczynska B, et al. TNF- $\alpha$ inhibits asbestos-induced cytotoxicity via a NF- $\mathrm{BB}$-dependent pathway, a possible mechanism for asbestos-induced oncogenesis. Proc Natl Acad Sci USA 2006; 103: 10397-10402.

22 Sekido Y. Molecular pathogenesis of malignant mesothelioma. Carcinogenesis 2013; 34: 1413-1419.

23 Sekido Y. Molecular biology of malignant mesothelioma. Environ Health Prev Med 2008; 13: 65-70.

24 Yang H, Rivera Z, Jube S, et al. Programmed necrosis induced by asbestos in human mesothelial cells causes high-mobility group box 1 protein release and resultant inflammation. Proc Natl Acad Sci USA 2010; 107: 12611-12616.

25 Jasani B, Gibbs A. Mesothelioma not associated with asbestos exposure. Arch Pathol Lab Med 2012; 136: 262-267.

26 Brenner J, Sordillo PP, Magill GB. Malignant mesothelioma in children: report of seven cases and review of the literature. Med Pediatr Oncol 1981; 9: 367-373.

27 Carbone M, Fisher S, Powers A, et al. New molecular and epidemiological issues in mesothelioma: role of SV40. J Cell Physiol 1999; 180: 167-172.

28 Kops SP. Oral polio vaccine and human cancer: a reassessment of SV40 as a contaminant based upon legal documents. Anticancer Res 2000; 20: 4745-4749. 
Carbone M, Pass HI, Rizzo P, et al. Simian virus 40-like DNA sequences in human pleural mesothelioma. Oncogene 1994; 9: 1781-1790.

Galateau-Salle F, Bidet P, Iwatsubo Y, et al. SV40-like DNA sequences in pleural mesothelioma, bronchopulmonary carcinoma, and non-malignant pulmonary diseases. J Pathol 1998; 184: 252-257.

Rotondo JC, Mazzoni E, Bononi I, et al. Association between simian virus 40 and human tumors. Front Oncol 2019; 9: 670 .

Testa JR, Carbone M, Hirvonen A, et al. A multi-institutional study confirms the presence and expression of simian virus 40 in human malignant mesotheliomas. Cancer Res 1998; 58: 4505-4509.

Cicala C, Pompetti F, Carbone M. SV40 induces mesotheliomas in hamsters. Am J Pathol 1993; 142: 1524-1533.

De Rienzo A, Tor M, Sterman DH, et al. Detection of SV40 DNA sequences in malignant mesothelioma specimens from the United States, but not from Turkey. J Cell Biochem 2002; 84: 455-459.

Hirvonen A, Mattson K, Karjalainen A, et al. Simian virus 40 (SV40)-like DNA sequences not detectable in Finnish mesothelioma patients not exposed to SV40-contaminated polio vaccines. Mol Carcinog 1999; 26: 93-99.

López-Ríos F, Illei PB, Rusch V, et al. Evidence against a role for SV40 infection in human mesotheliomas and high risk of false-positive PCR results owing to presence of SV40 sequences in common laboratory plasmids. Lancet 2004; 364: 1157-1166.

Austin MB, Fechner RE, Roggli VL. Pleural malignant mesothelioma following Wilms' tumor. Am J Clin Pathol 1986; 86: 227-230.

Cavazza A, Travis LB, Travis WD, et al. Post-irradiation malignant mesothelioma. Cancer 1996; 77: 1379-1385.

Teta MJ, Lau E, Sceurman BK, et al. Therapeutic radiation for lymphoma: risk of malignant mesothelioma. Cancer 2007; 109: 1432-1438.

Baris YI, Sahin AA, Ozesmi M, et al. An outbreak of pleural mesothelioma and chronic fibrosing pleurisy in the village of Karain/Urgüp in Anatolia. Thorax 1978; 33: 181-192.

Rohl AN, Langer AM, Moncure G, et al. Endemic pleural disease associated with exposure to mixed fibrous dust in Turkey. Science 1982; 216: 518-520.

Roushdy-Hammady I, Siegel J, Emri S, et al. Genetic-susceptibility factor and malignant mesothelioma in the Cappadocian region of Turkey. Lancet 2001; 357: 444-445.

Wagner JC, Skidmore JW, Hill RJ, et al. Erionite exposure and mesotheliomas in rats. Br J Cancer 1985; 51: 727-730.

Panou V, Gadiraju M, Wolin A, et al. Frequency of germline mutations in cancer susceptibility genes in malignant mesothelioma. J Clin Oncol 2018; 36: 2863-2871.

Carbone M, Gaudino G, Yang H. Recent insights emerging from malignant mesothelioma genome sequencing. J Thorac Oncol 2015; 10: 409-411.

Guo G, Chmielecki J, Goparaju C, et al. Whole-exome sequencing reveals frequent genetic alterations in BAP1, NF2, CDKN2A, and CUL1 in malignant pleural mesothelioma. Cancer Res 2015; 75: 264-269.

Hida T, Matsumoto S, Hamasaki M, et al. Deletion status of p16 in effusion smear preparation correlates with that of underlying malignant pleural mesothelioma tissue. Cancer Sci 2015; 106: 1635-1641.

Hmeljak J, Sanchez-Vega F, Hoadley KA, et al. Integrative molecular characterization of malignant pleural mesothelioma. Cancer Discov 2018; 8: 1548-1565.

Lo Iacono M, Monica V, Righi L, et al. Targeted next-generation sequencing of cancer genes in advanced stage malignant pleural mesothelioma: a retrospective study. J Thorac Oncol 2015; 10: 492-499.

Zehir A, Benayed R, Shah RH, et al. Mutational landscape of metastatic cancer revealed from prospective clinical sequencing of 10,000 patients. Nat Med 2017; 23: 703-713.

Metcalf RA, Welsh JA, Bennett WP, et al. p53 and Kirsten-ras mutations in human mesothelioma cell lines. Cancer Res 1992; 52: 2610-2615.

Tian K, Rajendran R, Doddananjaiah M, et al. Dynamics of DNA damage induced pathways to cancer. PLoS One 2013; 8: e72303.

Bott M, Brevet M, Taylor BS, et al. The nuclear deubiquitinase BAP1 is commonly inactivated by somatic mutations and 3p21.1 losses in malignant pleural mesothelioma. Nat Genet 2011; 43: 668-672.

Bueno R, Stawiski EW, Goldstein LD, et al. Comprehensive genomic analysis of malignant pleural mesothelioma identifies recurrent mutations, gene fusions and splicing alterations. Nat Genet 2016; 48: 407-416.

Singhi AD, Krasinskas AM, Choudry HA, et al. The prognostic significance of BAP1, NF2, and CDKN2A in malignant peritoneal mesothelioma. Modern Pathol 2016; 29: 14-24.

Jongsma J, van Montfort E, Vooijs M, et al. A conditional mouse model for malignant mesothelioma. Cancer Cell 2008; 13: 261-271.

Bowman R, Relan V, Hughes B. Medical management of mesothelioma. Aust Prescr 2011; 34: 144-147.

Segal A, Sterrett GF, Frost FA, et al. A diagnosis of malignant pleural mesothelioma can be made by effusion cytology: results of a 20 year audit. Pathology 2013; 45: 44-48.

Arnold DT, De Fonseka D, Perry S, et al. Investigating unilateral pleural effusions: the role of cytology Eur Respir J 2018; 52: 1801254.

S, Paterson S, Cartwright D, et al. Baseline predictors of negative and incomplete pleural cytology in patients with suspected pleural malignancy - data supporting 'Direct to LAT' in selected groups. Lung Cancer 2019; 133: 123-129.

Kindler HL, Ismaila N, Armato SG, et al. Treatment of malignant pleural mesothelioma: American Society of Clinical Oncology clinical practice guideline. J Clin Oncol 2018; 36: 1343-1373.

Scherpereel A, Opitz I, Berghmans T, et al. ERS/ESTS/EACTS/ESTRO guidelines for the management of malignant pleural mesothelioma. Eur Respir J 2020; 55: 1900953.

Bibby AC, Dorn P, Psallidas I, et al. ERS/EACTS statement on the management of malignant pleural effusions. Eur Respir J 2018; 52: 1800349.

Sconfienza LM, Mauri G, Grossi F, et al. Pleural and peripheral lung lesions: comparison of US- and CT-guided biopsy. Radiology 2013; 266: 930-935.

Hallifax RJ, Corcoran JP, Ahmed A, et al. Physician-based ultrasound-guided biopsy for diagnosing pleural disease. Chest 2014; 146: 1001-1006. 

diagnosis of malignant disease in pleural effusions: a randomised controlled trial. Lancet 2003; 361: 1326-1330.

67 Wijmans L, Baas P, Sieburgh TE, et al. Confocal laser endomicroscopy as a guidance tool for pleural biopsies in malignant pleural mesothelioma. Chest 2019; 156: 754-763.

68 DeFonsecka D. PET-CT in the undiagnosed effusion: results of the TARGET study, presented at the British Thoracic Society Winter Meeting December 4-6, 2019. Thorax 2019; 74: Suppl. 2.

69 Baas P, Fennell D, Kerr KM, et al. Malignant pleural mesothelioma: ESMO Clinical Practice Guidelines for diagnosis, treatment and follow-up. Ann Oncol 2015; 26: v31-v39.

70 Stewart D, Waller D, Edwards J, et al. Is there a role for pre-operative contrast-enhanced magnetic resonance imaging for radical surgery in malignant pleural mesothelioma? Eur J Cardiothorac Surg 2003; 24: 1019-1024.

71 Flores RM, Akhurst $\mathrm{T}$, Gonen $\mathrm{M}$, et al. Positron emission tomography defines metastatic disease but not locoregional disease in patients with malignant pleural mesothelioma. J Thorac Cardiovasc Surgery 2003; 126: 11-16.

72 Lee HY, Hyun SH, Lee KS, et al. Volume-based parameter of 18F-FDG PET/CT in malignant pleural mesothelioma: prediction of therapeutic response and prognostic implications. Ann Surg Oncol 2010; 17: 2787-2794.

73 Wilcox BE, Subramaniam RM, Peller PJ, et al. Utility of integrated computed tomography-positron emission tomography for selection of operable malignant pleural mesothelioma. Clin Lung Cancer 2009; 10: 244-248.

74 Rice D, Rusch V, Pass $\mathrm{H}$, et al. Recommendations for uniform definitions of surgical techniques for malignant pleural mesothelioma: a consensus report of the International Association for the Study of Lung Cancer International Staging Committee and the International Mesothelioma Interest Group. J Thorac Oncol 2011; 6: 1304-1312.

75 Lapidot M, Freyaldenhoven S, Bueno R. New concepts in the treatment of malignant pleural mesothelioma. J Thorac Dis 2018; 10: 1283-1285.

76 Treasure T, Lang-Lazdunski L, Waller D, et al. Extra-pleural pneumonectomy versus no extra-pleural pneumonectomy for patients with malignant pleural mesothelioma: clinical outcomes of the Mesothelioma and Radical Surgery (MARS) randomised feasibility study. Lancet Oncol 2011; 12: 763-772.

77 Taioli E, Wolf AS, Flores RM. Meta-analysis of survival after pleurectomy decortication versus extrapleural pneumonectomy in mesothelioma. Ann Thorac Surgery 2015; 99: 472-480.

78 Cao C, Tian D, Park J, et al. A systematic review and meta-analysis of surgical treatments for malignant pleural mesothelioma. Lung Cancer 2014; 83: 240-245.

79 Treasure T. Extrapleural pneumonectomy for malignant pleural mesothelioma: is this an operation that should now be consigned to history? Future Oncol 2015; 11: 7-10.

80 Schwartz RM, Lieberman-Cribbin W, Wolf A, et al. Systematic review of quality of life following pleurectomy decortication and extrapleural pneumonectomy for malignant pleural mesothelioma. BMC Cancer 2018; $18: 1188$.

81 Rintoul RC, Ritchie AJ, Edwards JG, et al. Efficacy and cost of video-assisted thoracoscopic partial pleurectomy versus talc pleurodesis in patients with malignant pleural mesothelioma (MesoVATS): an open-label, randomised, controlled trial. Lancet 2014; 384: 1118-1127.

82 Van Meerbeeck JP, Gaafar R, Manegold C, et al. Randomized phase III study of cisplatin with or without raltitrexed in patients with malignant pleural mesothelioma: an intergroup study of the European Organisation for Research and Treatment of Cancer Lung Cancer Group and the National Cancer Institute. J Clin Oncol 2005; 23: 6881-6889.

83 Mansfield AS, Symanowski JT, Peikert T. Systematic review of response rates of sarcomatoid malignant pleural mesotheliomas in clinical trials. Lung Cancer 2014; 86: 133-136.

84 Buikhuisen WA, Hiddinga BI, Baas P, et al. Second line therapy in malignant pleural mesothelioma: a systematic review. Lung Cancer 2015; 89: 223-231.

85 Jassem J, Ramlau R, Santoro A, et al. Phase III trial of pemetrexed plus best supportive care compared with best supportive care in previously treated patients with advanced malignant pleural mesothelioma. J Clin Oncol 2008; 26: $1698-1704$.

86 Nowak A, Kok P, Lesterhuis W, et al. DREAM - a phase 2 trial of durvalumab with first line chemotherapy in mesothelioma: final result. J Thorac Oncol 2018; 13: OA08.02.

87 Stephens RJ, Whiting C, Cowan KK, et al. Research priorities in mesothelioma: a James Lind Alliance Priority Setting Partnership. Lung Cancer 2015; 89: 175-180.

88 Hasegawa S, Okada M, Tanaka F, et al. Trimodality strategy for treating malignant pleural mesothelioma: results of a feasibility study of induction pemetrexed plus cisplatin followed by extrapleural pneumonectomy and postoperative hemithoracic radiation (Japan Mesothelioma Interest Group 0601 Trial). Int J Clin Oncol 2016; 21: 523-530.

89 Stahel RA, Riesterer O, Xyrafas A, et al. Neoadjuvant chemotherapy and extrapleural pneumonectomy of malignant pleural mesothelioma with or without hemithoracic radiotherapy (SAKK 17/04): a randomised, international, multicentre phase 2 trial. Lancet Oncol 2015; 16: 1651-1658.

90 MacLeod N, Price A, O’Rourke N, et al. Radiotherapy for the treatment of pain in malignant pleural mesothelioma: a systematic review. Lung Cancer 2014; 83: 133-138.

91 MacLeod N, Chalmers A, O’Rourke N, et al. Is radiotherapy useful for treating pain in mesothelioma?: a phase II trial. J Thorac Oncol 2015; 10: 944-950.

92 Bhatnagar R, Maskell N. Developing a 'pleural team' to run a reactive pleural service. Clin Med 2013; 13: 452-456.

93 Rahman NM, Pepperell J, Rehal S, et al. Effect of opioids $v s$ NSAIDs and larger $v s$ smaller chest tube size on pain control and pleurodesis efficacy among patients with malignant pleural effusion: the TIME1 randomized clinical trial. JAMA 2015; 314: 2641-2653.

94 Mercer RM, Macready J, Jeffries $\mathrm{H}$, et al. Clinically important associations of pleurodesis success in malignant pleural effusion: analysis of the TIME1 data set. Respirology 2020; 25: 750-755.

95 Taghizadeh N, Fortin M, Tremblay A. US hospitalizations for malignant pleural effusions: data from the 2012 national inpatient sample. Chest 2017; 151: 845-854.

96 Davies HE, Mishra EK, Kahan BC, et al. Effect of an indwelling pleural catheter $v s$ chest tube and talc pleurodesis for relieving dyspnea in patients with malignant pleural effusion: the TIME2 randomized controlled trial. JAMA 2012; 307: 2383-2389. 

hospitalization days in patients with malignant pleural effusion: the AMPLE randomized clinical trial. JAMA 2017; 318: 1903-1912.

98 Boshuizen RC, Vd Noort V, Burgers JA, et al. A randomized controlled trial comparing indwelling pleural catheters with talc pleurodesis (NVALT-14). Lung Cancer 2017; 108: 9-14.

99 Van Meter MEM, McKee KY, Kohlwes RJ. Efficacy and safety of tunneled pleural catheters in adults with malignant pleural effusions: a systematic review. J Gene Intern Med 2011; 26: 70-76.

100 Tremblay A, Mason C, Michaud G. Use of tunnelled catheters for malignant pleural effusions in patients fit for pleurodesis. Eur Respir J 2007; 30: 759-762.

101 Asciak R, Hallifax RJ, Mercer RM, et al. The hospital and patient burden of indwelling pleural catheters: a retrospective case series of 210 indwelling pleural catheter insertions. Respiration 2019; 97: 70-77.

102 Cameron K, Teodoro D, Kasis A, et al. Development and maintenance of a pleural disease service: role of the "pleurologist". Semin Respir Crit Care Med 2019; 40: 297-304.

103 Wahidi MM, Reddy C, Yarmus L, et al. Randomized trial of pleural fluid drainage frequency in patients with malignant pleural effusions. The ASAP trial. Am J Respir Crit Care Med 2017; 195: 1050-1057.

104 Muruganandan S, Azzopardi M, Fitzgerald DB, et al. Aggressive versus symptom-guided drainage of malignant pleural effusion via indwelling pleural catheters (AMPLE-2): an open-label randomised trial. Lancet Respir 2018; 6: $671-680$.

105 Bhatnagar R, Keenan EK, Morley AJ, et al. Outpatient talc administration by indwelling pleural catheter for malignant effusion. N Engl J Med 2018; 378: 1313-1322.

106 Hassan M, Mercer RM, Maskell NA, et al. Survival in patients with malignant pleural effusion undergoing talc pleurodesis. Lung Cancer 2019; 137: 14-18.

107 Brims F, Gunatilake S, Lawrie I, et al. Early specialist palliative care on quality of life for malignant pleural mesothelioma: a randomised controlled trial. Thorax 2019; 74: 354-361. 